\title{
Kinetic analysis of intense sheet beam stability properties for uniform phase-space density
}

\author{
Edward A. Startsev and Ronald C. Davidson \\ Plasma Physics Laboratory, Princeton University, Princeton, New Jersey 08543
}

(Received 3 December 2002; published 17 April 2003)

\begin{abstract}
This paper makes use of the Vlasov-Maxwell equations to investigate collective excitations in an intense sheet beam, infinite in the $y$ and $z$ directions, propagating in the $z$ direction with directed kinetic energy $\left(\gamma_{b}-1\right) m_{b} c^{2}$. The beam is confined in the $x$ direction by the smooth-focusing force $\vec{F}_{\text {foc }}=-\gamma_{b} m_{b} \omega_{\beta \perp}^{2} x \overrightarrow{\boldsymbol{e}}_{x}$, and perfectly conducting walls are located at $x= \pm x_{w}$. A self-consistent water bag equilibrium $f_{b}^{0}$ satisfying the steady-state $(\partial / \partial t=0)$ Vlasov-Maxwell equations is shown to be exactly solvable for the beam density $n_{b}^{0}(x)$ and electrostatic potential $\phi^{0}(x)$. A closed Schrödinger-like eigenvalue equation is derived, assuming small-amplitude perturbations $\left(\delta f_{b}, \delta \phi\right)$ about the selfconsistent water bag equilibrium, and the eigenfrequency spectrum is shown to be purely real. The WKB approximation is employed to determine the eigenfrequency spectrum as a function of the normalized beam intensity $s_{b}=\hat{\omega}_{p b}^{2} / \gamma_{b}^{2} \omega_{\beta \perp}^{2}$, where $\hat{\omega}_{p b}^{2}=4 \pi \hat{n}_{b} e_{b}^{2} / \gamma_{b} m_{b}$ and $\hat{n}_{b}=n_{b}(x=0)$ is the on-axis number density of beam particles.
\end{abstract}

DOI: 10.1103/PhysRevSTAB.6.044401

PACS numbers: 29.27.-a, 41.75.-i

\section{INTRODUCTION}

High-intensity accelerators, transport systems, and storage rings [1-5] have a wide range of applications ranging from basic research in high energy and nuclear physics, to applications such as heavy ion fusion, spallation neutron sources, tritium production, and nuclear waste transmutation. Of particular importance at the high beam currents and charge densities of practical interest are the effects off the intense self-fields produced by the beam space charge and current on determining detailed equilibrium, stability, and transport properties. In general, a complete description of collective processes in intense charged particle beams is provided by the nonlinear Vlasov-Maxwell equations [1-3] for the selfconsistent evolution of the beam distribution function $f_{b}(\vec{x}, \vec{p}, t)$, and the electric and magnetic fields, $\vec{E}(\vec{x}, t)$ and $\vec{B}(\vec{x}, t)$. While considerable progress has been made in the analytical and numerical simulation studies of intense beam propagation [6-35], the effects of finite geometry and intense self-fields often make it difficult to obtain detailed predictions of beam equilibrium, stability, and transport properties based on the Vlasov-Maxwell equations. For example, the only equilibrium distribution function for which detailed analytical estimates of beam stability properties have been carried out at high beam intensity is the so-called Kapchinskij-Vladimirskij distribution function [1,32-36]. Such a distribution function, due to its highly inverted population in phase space, is of very limited practical interest. Therefore, an investigation of intense beam stability properties in circumstances where solutions are analytically tractable remains a problem of considerable fundamental importance for elucidating the underlying physics, which we examine in this paper for an intense sheet beam [6] with uniform phase-space density propagating through a smoothfocusing field.
The present analysis makes use of the Vlasov-Maxwell equations [1] to investigate collective excitations in an intense sheet beam [6], infinite in the $y$ and $z$ directions, propagating in the $z$ direction with directed kinetic energy $\left(\gamma_{b}-1\right) m_{b} c^{2}$. The beam is confined in the $x$ direction by the smooth-focusing force $\vec{F}_{\text {loc }}=-\gamma_{b} m_{b} \omega_{\beta \perp}^{2} x \vec{e}_{x}$, and perfectly conducting walls are located at $x= \pm x_{w}$. A self-consistent water bag equilibrium $f_{b}^{0}\left(x, p_{x}\right)$, with uniform phase-space density, satisfying the steady-state $(\partial / \partial t=0)$ Vlasov-Maxwell equations is shown to be exactly solvable for the beam density $n_{b}^{0}(x)$ and electrostatic potential $\phi^{0}(x)$. A closed Schrödinger-like eigenvalue equation is derived, assuming small-amplitude perturbations $\left(\delta f_{b}, \delta \phi\right)$ about the self-consistent water bag equilibrium, and the eigenfrequency spectrum is shown to be purely real. The WKB approximation is employed to determine the eigenfrequency spectrum as a function of the normalized beam intensity $s_{b}=\hat{\omega}_{p b}^{2} / \gamma_{b}^{2} \omega_{\beta \perp}^{2}$, where $\hat{\omega}_{p b}^{2}=4 \pi \hat{n}_{b} e_{b}^{2} / \gamma_{b} m_{b}$ is the relativistic plasma frequency squared, and $\hat{n}_{b}=n_{b}(x=0)$ is the on-axis number density of beam particles. Most remarkably, for perturbations about a water bag equilibrium with uniform phase-space density, the kinetic analysis leads to an eigenvalue equation that is analytically tractable over a wide range of system parameters, with eigenfrequency solutions determined in terms of elliptic integrals.

The organization of this paper is the following. The theoretical model and assumptions are described in Sec. II, and in Sec. III detailed equilibrium properties are calculated for a water bag equilibrium distribution $f_{b}\left(x, p_{x}\right)$ with uniform phase-space density [Eq. (8)]. The linearized Vlasov-Maxwell equations are investigated in Sec. IV for small-amplitude perturbations $\left(\delta f_{b}, \delta \phi\right)$ about the water bag equilibrium $f_{b}\left(x, p_{x}\right)$, which leads to the eigenvalue equation (48) for the perturbed electric field $\delta E_{x}$. Finally, in Sec. V, the eigenvalue equation is 
investigated analytically and numerically, and the eigenfrequencies $\left\{\omega_{m}^{2}\right\}$ are determined over the entire range of beam intensities, ranging from low beam intensity $\left(s_{b} \ll 1\right)$ to a space-charge-dominated beam $\left(s_{b} \rightarrow 1\right)$, where $s_{b}=\hat{\omega}_{p b}^{2} / \gamma_{b}^{2} \omega_{\beta \perp}^{2}$ is the normalized beam intensity.

\section{THEORETICAL MODEL AND ASSUMPTIONS}

We consider an intense sheet beam, made up of particles with charge $e_{b}$ and rest mass $m_{b}$, which propagates in the $z$ direction with directed kinetic energy $\left(\gamma_{b}-1\right) m_{b} c^{2}$ and average axial velocity $V_{b}=\beta_{b} c=$ const. Here, $\gamma_{b}=\left(1-\beta_{b}^{2}\right)^{-1 / 2}$ is the relativistic mass factor, $c$ is the speed of light in vacuo, and the beam is assumed to be uniform in the $y$ and $z$ directions with $\partial / \partial y=0=\partial / \partial z$. The beam is centered in the $x$ direction at $x=0$, and transverse confinement is provided by an applied focusing force, $F_{x}^{\text {foc }}=-\gamma_{b} m_{b} \omega_{\beta \perp}^{2} x$, with $\omega_{\beta \perp}^{2}=$ const in the smooth-focusing approximation. The transverse dimension of the sheet beam is denoted by $2 x_{b}$, and planar, perfectly conducting walls are located at $x= \pm x_{w}$. Finally, the particle motion in the beam frame is assumed to be nonrelativistic.

Within the context of the assumptions summarized above, the nonlinear Vlasov-Maxwell equations [1] provide a complete description of the evolution of the distribution function $f_{b}\left(x, p_{x}, t\right)$ in the phase space $\left(x, p_{x}\right)$ due to the interaction of the beam particles with the applied focusing field and the self-generated electric and magnetic fields, $E_{x}^{s}(x, t)=-(\partial / \partial x) \phi(x, t)$ and $B_{y}^{s}(x, t)=-\beta_{b}(\partial / \partial x) \phi(x, t)$. Here, $\phi(x, t)$ is the spacecharge potential determined from Poisson's equation, and $A_{z}(x, t)=\beta_{b} \phi(x, t)$ has been assumed [1,37]. It is convenient to introduce the effective potential $\psi(x, t)$ defined by

$$
\psi(x, t)=\frac{1}{2} \gamma_{b} m_{b} \omega_{\beta \perp}^{2} x^{2}+\frac{1}{\gamma_{b}^{2}} e_{b} \phi(x, t)
$$

Then the Vlasov-Maxwell equations describing the selfconsistent nonlinear evolution of $f_{b}\left(x, p_{x}, t\right)$ and $\psi(x, t)$ can be expressed as $[1,6]$

$$
\left(\frac{\partial}{\partial t}+v_{x} \frac{\partial}{\partial x}-\frac{\partial \psi}{\partial x} \frac{\partial}{\partial p_{x}}\right) f_{b}=0
$$

and

$$
\frac{\partial^{2} \psi}{\partial x^{2}}=\gamma_{b} m_{b} \omega_{\beta \perp}^{2}-\frac{4 \pi e_{b}^{2}}{\gamma_{b}^{2}} \int_{-\infty}^{\infty} d p_{x} f_{b}
$$

Here, $v_{x}=p_{x} / \gamma_{b} m_{b}$ is the transverse particle velocity,

$$
n_{b}(x, t)=\int_{-\infty}^{\infty} d p_{x} f_{b}\left(x, p_{x}, t\right)
$$

is the number density of the beam particles, and Eq. (3) follows directly from Eq. (1) and Poisson's equation $\partial^{2} \phi / \partial x^{2}=-4 \pi e_{b} n_{b}$.

Equations (2) and (3) provide a complete nonlinear description of collective interactions in an intense sheet beam assuming one-dimensional spatial variations in the $x$ direction. In general, for perfectly conducting planar walls located at $x= \pm x_{w}$, Eqs. (2) and (3) are to be solved subject to the boundary conditions $\phi\left(x= \pm x_{w}, t\right)=$ const.

\section{SHEET BEAM EQUILIBRIUM WITH UNIFORM PHASE-SPACE DENSITY}

Under steady-state conditions $(\partial / \partial t=0)$, Eqs. (2) and (3) support a broad class of equilibrium solutions of the form [1]

$$
f_{b}^{0}\left(x, p_{x}\right)=F_{b}\left(H_{\perp}\right)
$$

where

$$
H_{\perp}=\frac{p_{x}^{2}}{2 \gamma_{b} m_{b}}+\psi^{0}(x)
$$

Here, the transverse Hamiltonian $H_{\perp}$ is an exact singleparticle constant of the motion in the equilibrium field configuration, and $\psi^{0}(x)$ is determined self-consistently in terms of the number density $n_{b}^{0}(x)=\int_{-\infty}^{\infty} d p_{x} F_{b}\left(H_{\perp}\right)$ from

$$
\frac{\partial^{2} \psi^{0}}{\partial x^{2}}=\gamma_{b} m_{b} \omega_{\beta \perp}^{2}-\frac{4 \pi e_{b}^{2}}{\gamma_{b}^{2}} \int_{-\infty}^{\infty} d p_{x} F_{b}\left(H_{\perp}\right),
$$

where $H_{\perp}$ is defined in Eq. (6). In general, depending on the choice of equilibrium distribution $F_{b}\left(H_{\perp}\right)$, Eq. (7) is a nonlinear differential equation for the effective potential $\psi^{0}(x)=\gamma_{b} m_{b} \omega_{\beta \perp}^{2} x^{2} / 2+\left(e_{b} / \gamma_{b}^{2}\right) \phi^{0}(x)$.

As an equilibrium example that is analytically tractable, and accessible to detailed stability analysis (Sec. IV), we consider the choice of distribution function

$$
F_{b}\left(H_{\perp}\right)=\frac{\hat{n}_{b}}{\left(8 \gamma_{b} m_{b} \hat{H}_{\perp}\right)^{1 / 2}} \Theta\left(H_{\perp}-\hat{H}_{\perp}\right),
$$

where $\hat{n}_{b}$ and $\hat{H}_{\perp}$ are positive constants. Here, $\Theta(x)$ is the Heaviside step function defined by $\Theta(x)=1$ for $x<0$, and $\Theta(x)=0$ for $x>0$. For the choice of distribution function in Eq. (8), note that the phase-space density $f_{b}^{0}\left(x, p_{x}\right)=F_{b}\left(H_{\perp}\right)$ is constant for $H_{\perp}<\hat{H}_{\perp}$, and equal to zero for $H_{\perp}>\hat{H}_{\perp}$. Without loss of generality, we take the zero of potential to correspond to $\psi^{0}(x=0)=0$. Because $\partial F_{b}\left(H_{\perp}\right) / \partial H_{\perp} \leq 0$ for the choice of distribution function in Eq. (8), the water bag equilibrium described by Eq. (8) is expected to give rise to stable collective oscillations for small-amplitude perturbations [21]. By contrast, distributions with $\partial F_{b}\left(H_{\perp}\right) / \partial H_{\perp}>0$ over a sufficiently large region of phase space may be unstable, because $\partial F_{b}\left(H_{\perp}\right) / \partial H_{\perp}>0$ is a necessary condition for instability $[1,21]$. 
From Eq. (8), the particle motion is constrained to $H_{\perp}<\hat{H}_{\perp}$, or equivalently, $p_{x}^{2}<\left(2 \gamma_{b} m_{b} \hat{H}_{\perp}\right)(1-$ $\left.\psi^{0} / \hat{H}_{\perp}\right)$. Evaluating the number density $n_{b}^{0}(x)=$ $\int_{-\infty}^{\infty} d p_{x} F_{b}\left(H_{\perp}\right)$, we readily obtain

$$
n_{b}^{0}(x)= \begin{cases}\hat{n}_{b}\left[1-\psi^{0}(x) / \hat{H}_{\perp}\right]^{1 / 2}, & -x_{b}<x<x_{b}, \\ 0, & |x|>x_{b} .\end{cases}
$$

Here, the location of the beam edge $\left(x= \pm x_{b}\right)$ is determined from

$$
\psi^{0}\left(x= \pm x_{b}\right)=\hat{H}_{\perp} .
$$

Because $\psi^{0}(x=0)=0$ is assumed, note from Eq. (9) that $\hat{n}_{b}=n_{b}^{0}(x=0)=$ const is the number density of beam particles at $x=0$. Moreover, substituting Eq. (9) into Poisson's equation (7), the physically acceptable solution for $\psi^{0}(x)$ increases monotonically from $\psi^{0}(x=0)=0$ until the value $\psi^{0}\left(x= \pm x_{b}\right)=\hat{H}_{\perp}>0$ in Eq. (10) is achieved. Note from Eq. (9) that the number density of beam particles decreases monotonically from $n_{b}^{0}(x=0)=\hat{n}_{b}$ to $n_{b}^{0}\left(x= \pm x_{b}\right)=0$ at the beam edges.

It is useful to introduce the normalized beam intensity $s_{b}$ and effective Debye length $\lambda_{D}$ defined by

$$
s_{b}=\frac{\hat{\omega}_{p b}^{2}}{\gamma_{b}^{2} \omega_{\beta \perp}^{2}}, \quad \lambda_{D}^{2}=\frac{\gamma_{b}^{3} \hat{H}_{\perp}}{4 \pi \hat{n}_{b} e_{b}^{2}}=\frac{1}{2} \frac{\gamma_{b}^{2} \hat{\boldsymbol{v}}_{0}^{2}}{\hat{\boldsymbol{\omega}}_{p b}^{2}} .
$$

Here, $\hat{\boldsymbol{\omega}}_{p b}=\left(4 \pi \hat{n}_{b} e_{b}^{2} / \gamma_{b} m_{b}\right)^{1 / 2}$ is the relativistic plasma frequency at $x=0$, and $\hat{\boldsymbol{v}}_{0}=\left(2 \hat{H}_{\perp} / \gamma_{b} m_{b}\right)^{1 / 2}$ is the maximum speed of a particle with energy $\hat{H}_{\perp}$ as it passes through $x=0$. Substituting Eq. (9) into Eq. (7) then gives

$$
\frac{\partial^{2}}{\partial x^{2}}\left(\frac{\psi^{0}(x)}{\hat{H}_{\perp}}\right)=\frac{1}{\lambda_{D}^{2}}\left(\frac{1}{s_{b}}-\left[1-\frac{\psi^{0}(x)}{\hat{H}_{\perp}}\right]^{1 / 2}\right)
$$

in the beam interior $\left(-x_{b}<x<x_{b}\right)$. Equation (12) is to be integrated subject to the boundary conditions $\left[\psi^{0}\right]_{x=0}=0=\left[\partial \psi^{0} / \partial x\right]_{x=0}$. For physically acceptable solutions to Eq. (12), the condition $\left[\partial^{2} \psi^{0} / \partial x^{2}\right]_{x=0}>0$ imposes the requirement that $s_{b}$ lies in the interval

$$
0<s_{b}<1 \text {, }
$$

where $s_{b}=\hat{\omega}_{p b}^{2} / \gamma_{b}^{2} \omega_{\beta \perp}^{2}$ is the normalized beam inten- sity. The reason for the requirement $\left[\partial^{2} \psi^{0} / \partial x^{2}\right]_{x=0}>0$ (which corresponds to $0<s_{b}<1$ ) can be seen as follows. If we assume that $\left[\partial^{2} \psi^{0} / \partial x^{2}\right]_{x=0}<0$, then it follows from Eq. (12) and $\left[\psi^{0}\right]_{x=0}=0$ that $s_{b}>1$. Integration of Eq. (12) for $s_{b}>1$ then shows that $\psi^{0}(x)<0$ for $|x|>0$, corresponding to a nonconfined density profile with monotonically increasing $n_{b}(x)$ for $|x|>0$.

In Eq. (13), the regime $s_{b} \ll 1$ corresponds to a low-intensity, emittance-dominated beam, whereas the regime $s_{b} \rightarrow 1$ corresponds to a low-emittance, spacecharge-dominated beam. In solving Eq. (12), it is convenient to introduce the dimensionless variables

$$
X=\frac{x}{\lambda_{D}}, \quad \hat{\psi}^{0}(X)=\frac{\psi^{0}(x)}{\hat{H}_{\perp}} .
$$

Substituting Eq. (14) into Eq. (12), integrating once, and enforcing $\left[\psi^{0}\right]_{x=0}=0=\left[\partial \psi^{0} / \partial x\right]_{x=0}$, gives

$$
\frac{1}{2}\left(\frac{d \hat{\psi}^{0}}{d X}\right)^{2}=\frac{1}{s_{b}} \hat{\psi}^{0}+\frac{2}{3}\left[\left(1-\hat{\psi}^{0}\right)^{3 / 2}-1\right]
$$

in the interval $-x_{b} / \lambda_{D} \leq X \leq x_{b} / \lambda_{D}$.

Equation (15) can be integrated exactly to determine $X$ as a function of $\left(1-\hat{\psi}^{0}\right)^{1 / 2}=n_{b}^{0}(X) / \hat{n}_{b}$ [see Eq. (9)]. We express $X=\int_{0}^{\hat{\psi}^{0}} d \hat{\psi}^{0} /\left(d \hat{\psi}^{0} / d X\right)$, and make use of Eq. (15). This gives

$$
X=\int_{0}^{\hat{\psi}^{0}} \frac{d \hat{\psi}^{0}}{2^{1 / 2}\left[\hat{\psi}^{0} / s_{b}-2 / 3+(2 / 3)\left(1-\hat{\psi}^{0}\right)^{3 / 2}\right]^{1 / 2}} .
$$

Changing variables to $z=\left(1-\hat{\psi}^{0}\right)^{1 / 2}$ or $\hat{\psi}^{0}=1-z^{2}$, Eq. (16) becomes

$$
X=3^{1 / 2} \int_{\left(1-\hat{\psi}^{0}\right)^{1 / 2}}^{1} \frac{z d z}{\left[(1-z)\left(a^{+}-z\right)\left(z-a^{-}\right)\right]^{1 / 2}},
$$

where $a^{+}$and $a^{-}$are defined by

$$
a^{ \pm}=\frac{1}{4 s_{b}}\left\{3-2 s_{b} \pm\left[3\left(3+4 s_{b}-4 s_{b}^{2}\right)\right]^{1 / 2}\right\} .
$$

Carrying out the integration in Eq. (17), we obtain

$$
X=\frac{2 \times 3^{1 / 2}}{\left(a^{+}-a^{-}\right)^{1 / 2}}\left\{\left(1-a^{+}\right) \Pi\left(\arcsin \kappa^{-1}\left[\frac{1-\left(1-\hat{\psi}^{0}\right)^{1 / 2}}{a^{+}-\left(1-\hat{\psi}^{0}\right)^{1 / 2}}\right]^{1 / 2}, \kappa^{2}, \kappa\right)+a^{+} F\left(\arcsin \kappa^{-1}\left[\frac{1-\left(1-\hat{\psi}^{0}\right)^{1 / 2}}{a^{+}-\left(1-\hat{\psi}^{0}\right)^{1 / 2}}\right]^{1 / 2}, \kappa\right)\right\},
$$

where $\kappa$ is defined by

$$
\kappa=\left(\frac{1-a^{+}}{a^{+}-a^{-}}\right)^{1 / 2}
$$

In Eq. (19), $F(\alpha, \kappa)$ is the elliptic integral of the first kind, and $\Pi(\alpha, n, \kappa)$ is the elliptic integral of the third kind [38]. Moreover, the solution in Eq. (19) is applicable in the beam interior corresponding to $-x_{b} / \lambda_{D}<X<x_{b} / \lambda_{D}$ and $0<\hat{\psi}^{0}(X)<1$.
Several points are noteworthy from the solution in Eq. (19). First, the parameters $\kappa$ and $a^{ \pm}$depend only on the value of the normalized beam intensity $s_{b}=$ $\hat{\boldsymbol{\omega}}_{p b}^{2} / \gamma_{b}^{2} \omega_{\beta \perp}^{2}$. Second, Eq. (19) gives a closed expression for $X=x / \lambda_{D}$ in terms of the normalized density profile $n_{b}^{0}(X) / \hat{n}_{b}=\left(1-\hat{\psi}^{0}\right)^{1 / 2}=\left[1-\psi^{0}(x) / \hat{H}_{\perp}\right]^{1 / 2}$. Finally, the condition $\hat{\psi}^{0}\left(X= \pm x_{b} / \lambda_{D}\right)=1$ determines the location of the beam edge at $x= \pm x_{b}$. From Eq. (19), we 
obtain for $x_{b}$

$$
\begin{array}{r}
\frac{x_{b}}{\lambda_{D}}=\frac{2 \times 3^{1 / 2}}{\left(a^{+}-a^{-}\right)^{1 / 2}}\left\{\left(1-a^{+}\right) \Pi\left[\arcsin \left(\kappa^{2} a^{+}\right)^{-1 / 2}, \kappa^{2}, \kappa\right]\right. \\
\left.+a^{+} F\left[\arcsin \left(\kappa^{2} a^{+}\right)^{-1 / 2}, \kappa\right]\right\} . \quad(21)
\end{array}
$$

Equation (21) gives a closed expression for $x_{b} / \lambda_{D}$ in terms of the normalized beam intensity $s_{b}$ for the choice of equilibrium distribution function $F_{b}\left(H_{\perp}\right)$ in Eq. (8). Other properties of the beam equilibrium can also be calculated. For example, the areal density of the beam particles, $N_{b}=\int_{-x_{b}}^{x_{b}} d x n_{b}^{0}(x)$, for the density profile in Eq. (9) can be expressed as

$$
N_{b}=2 \hat{n}_{b} \int_{0}^{x_{b}} d x\left[1-\psi^{0}(x) / \hat{H}_{\perp}\right]^{1 / 2} .
$$

Some algebraic manipulation that makes use of Eqs. (14), (15), and (22) gives

$$
\frac{N_{b}}{2 \hat{n}_{b} x_{b}}=3^{1 / 2} \frac{\lambda_{D}}{x_{b}} \int_{0}^{1} \frac{z^{2} d z}{\left[(1-z)\left(a^{+}-z\right)\left(z-a^{-}\right)\right]^{1 / 2}},
$$

where $x_{b} / \lambda_{D}$ is defined in terms of $s_{b}$ in Eq. (21). Note from Eq. (23) that $N_{b} / 2 \hat{n}_{b} x_{b}$ depends only on the dimensionless intensity parameter $s_{b}$.

Equations (19) - (23) can be used to determine (numerically) universal plots of the normalized density profile $2 x_{b} n_{b}^{0}(x) / N_{b}$ versus $x / x_{b}$ for different values of the normalized beam intensity $\left(s_{b}\right)$ and layer thickness $\left(x_{b} / \lambda_{D}\right)$. Typical profiles are illustrated in Fig. 1 for values of $s_{b}$ ranging from $s_{b}=0.2$ to $s_{b}=0.999999$. For a lowintensity, high-emittance beam with $s_{b} \ll 1$, the solution for $n_{b}^{0}(x)$ obtained from Eq. (19) can be approximated by

$$
n_{b}^{0}(x)=\hat{n}_{b}\left(1-\frac{x^{2}}{x_{b}^{2}}\right)^{1 / 2}
$$

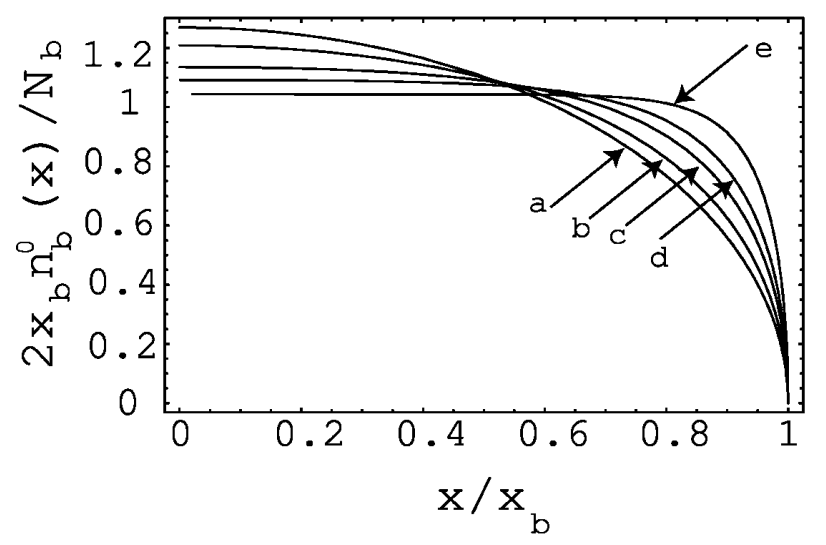

FIG. 1. Plots of the normalized density profile $2 x_{b} n_{b}^{0}(x) / N_{b}$ versus $x / x_{b}$ for different values of the normalized beam intensity $s_{b}$ corresponding to $(a) s_{b}=0.2,(b) s_{b}=0.9,(c) s_{b}=$ $0.99,(d) s_{b}=0.999$, and $(e) s_{b}=0.999999$.

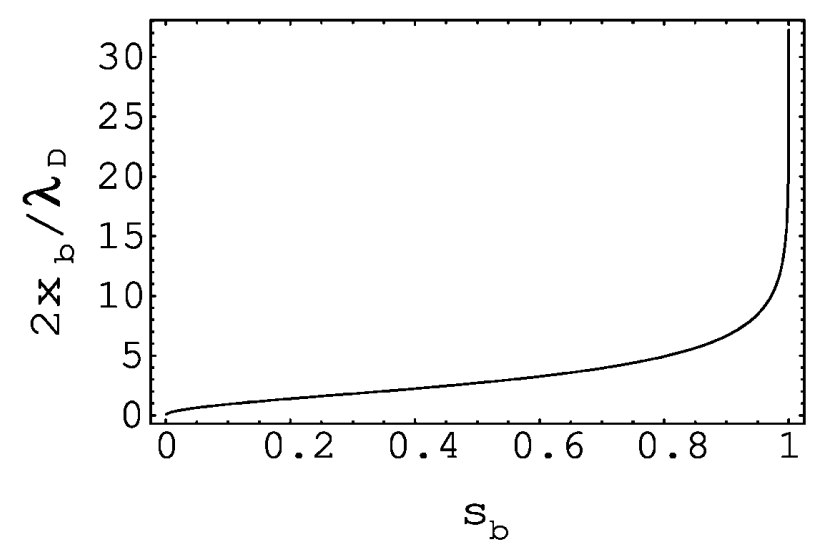

FIG. 2. Plot of the normalized layer thickness $2 x_{b} / \lambda_{D}$ versus the normalized beam intensity $s_{b}$ obtained from Eq. (21).

for $-x_{b}<x<x_{b}$. Here, $N_{b}=\int_{-x_{b}}^{x_{b}} d x n_{b}^{0}(x)=\hat{n}_{b} x_{b} / 2$, where $x_{b}^{2}=2 \hat{H}_{\perp} / \gamma_{b} m_{b} \omega_{\beta \perp}^{2}$. On the other hand, for a low-emittance, high-intensity beam with $s_{b} \rightarrow 1$ and $\lambda_{D} \rightarrow 0$, we obtain from Eq. (19) the step-function density profile

$$
n_{b}^{0}(x)= \begin{cases}\hat{n}_{b}=\text { const, } & -x_{b}<x<x_{b}, \\ 0, & |x|>x_{b},\end{cases}
$$

where $x_{b}=2 \pi N_{b} e_{b}^{2} / \gamma_{b}^{3} m_{b} \omega_{\beta \perp}^{2}$ is the half-thickness of the layer in the high-intensity limit, and $N_{b}=2 x_{b} \hat{n}_{b}$. Shown in Fig. 2 is a plot of the normalized layer thickness $2 x_{b} / \lambda_{D}$ versus $s_{b}$ obtained from Eq. (21).

Finally, other equilibrium properties can readily be calculated for the choice of distribution function in Eq. (8). For example, defining the equilibrium transverse pressure by $P_{b}^{0}(x)=\int_{-\infty}^{\infty} d p_{x}\left(p_{x}^{2} / \gamma_{b} m_{b}\right) f_{b}^{0}$, we readily obtain

$$
P_{b}^{0}(x)=\frac{4}{3} \hat{n}_{b} \hat{H}_{\perp}\left[1-\frac{\psi^{0}(x)}{\hat{H}_{\perp}}\right]^{3 / 2}
$$

in the beam interior $\left(-x_{b}<x<x_{b}\right)$. Comparing Eqs. (9) and (26), we note that $P_{b}^{0}(x)=\mathrm{const}\left[n_{b}^{0}(x)\right]^{3}$, corresponding to a triple-adiabatic pressure relation.

\section{LINEARIZED VLASOV-MAXWELL EQUATIONS}

In this section, we make use of Eqs. (2) and (3) to investigate the evolution of small-amplitude perturbations about the equilibrium distribution $f_{b}^{0}\left(x, p_{x}\right)=$ $F_{b}\left(H_{\perp}\right)$ and effective potential $\psi^{0}(x)$ described by Eqs. (8) and (12). Expanding $f_{b}\left(x, p_{x}, t\right)=F_{b}\left(H_{\perp}\right)+$ $\delta f_{b}\left(x, p_{x}, t\right)$ and $\psi(x, t)=\psi^{0}(x)+\delta \psi(x, t)$, the linearized Vlasov-Maxwell equations can be expressed as $[1,6]$

$$
\left(\frac{\partial}{\partial t}+v_{x} \frac{\partial}{\partial x}-\frac{\partial \psi^{0}}{\partial x} \frac{\partial}{\partial p_{x}}\right) \delta f_{b}=v_{x} \frac{\partial \delta \psi}{\partial x} \frac{\partial F_{b}}{\partial H_{\perp}},
$$

and 


$$
\frac{\partial^{2}}{\partial x^{2}} \delta \psi=-\frac{4 \pi e_{b}^{2}}{\gamma_{b}^{2}} \delta n_{b},
$$

where $\delta n_{b}(x, t)=\int_{-\infty}^{\infty} d p_{x} \delta f_{b}$ is the perturbed number density of beam particles. Here, use has been made of Eq. (6) and $\left(\partial / \partial p_{x}\right) F_{b}\left(H_{\perp}\right)=v_{x}\left(\partial / \partial H_{\perp}\right) F_{b}\left(H_{\perp}\right)$, where $v_{x}=p_{x} / \gamma_{b} m_{b}$ is the particle velocity. In analyzing Eqs. (27) and (28), it is convenient to change variables from $\left(x, p_{x}, t\right)$ to the new variables $\left(x^{\prime}, H_{\perp}, \tau\right)$ defined by

$$
\begin{gathered}
x^{\prime}=x, \quad \tau=t, \\
H_{\perp}=\frac{1}{2 \gamma_{b} m_{b}} p_{x}^{2}+\psi^{0}(x) .
\end{gathered}
$$

In this case, derivatives transform according to

$$
\begin{aligned}
\frac{\partial}{\partial t} & =\frac{\partial}{\partial \tau}, \\
\frac{\partial}{\partial x} & =\frac{\partial}{\partial x^{\prime}}+\frac{\partial \psi^{0}}{\partial x^{\prime}} \frac{\partial}{\partial H_{\perp}}, \\
\frac{\partial}{\partial p_{x}} & =v_{x} \frac{\partial}{\partial H_{\perp}}
\end{aligned}
$$

where

$$
v_{x}= \pm v\left(H_{\perp}, x^{\prime}\right) \equiv \pm\left(\frac{2 H_{\perp}}{\gamma_{b} m_{b}}\right)^{1 / 2}\left[1-\frac{\psi^{0}\left(x^{\prime}\right)}{H_{\perp}}\right]^{1 / 2} \text {. }
$$

Substituting Eqs. (29) and (30) into Eqs. (27) and (28) gives for the evolution of the perturbations $\delta f_{b}\left(x^{\prime}, H_{\perp}, \tau\right)$ and $\delta \psi\left(x^{\prime}, \tau\right)$,

$$
\begin{gathered}
\left(\frac{\partial}{\partial \tau}+v_{x} \frac{\partial}{\partial x^{\prime}}\right) \delta f_{b}=v_{x} \frac{\partial \delta \psi}{\partial x^{\prime}} \frac{\partial F_{b}}{\partial H_{\perp}}, \\
\frac{\partial^{2}}{\partial x^{\prime 2}} \delta \psi=-\frac{4 \pi e_{b}^{2}}{\gamma_{b}^{2}} \delta n_{b} .
\end{gathered}
$$

In Eq. (32), $v_{x}=+v\left(H_{\perp}, x^{\prime}\right)$ for the forward-moving particles with $v_{x}>0$, and $v_{x}=-v\left(H_{\perp}, x^{\prime}\right)$ for the backward-moving particles with $v_{x}<0$.

In analyzing Eqs. (32) and (33), it is convenient to express

$$
\begin{aligned}
\delta f_{b}\left(x^{\prime}, H_{\perp}, \tau\right)= & f_{b}^{+}\left(x^{\prime}, H_{\perp}, \tau\right) \Theta\left(-v_{x}\right) \\
& +f_{b}^{-}\left(x^{\prime}, H_{\perp}, \tau\right) \Theta\left(v_{x}\right),
\end{aligned}
$$

where $f_{b}^{+}\left(x^{\prime}, H_{\perp}, \tau\right)$ is the perturbed distribution of particles with $v_{x}>0$, and $f_{b}^{-}\left(x^{\prime}, H_{\perp}, \tau\right)$ is the perturbed distribution of particles with $v_{x}<0$. Substituting Eq. (34) into Eq. (32) gives

$$
\begin{gathered}
\left(\frac{\partial}{\partial \tau}+v \frac{\partial}{\partial x^{\prime}}\right) f_{b}^{+}=v \frac{\partial \delta \psi}{\partial x^{\prime}} \frac{\partial F_{b}}{\partial H_{\perp}}, \\
\left(\frac{\partial}{\partial \tau}-v \frac{\partial}{\partial x^{\prime}}\right) f_{b}^{-}=-v \frac{\partial \delta \psi}{\partial x^{\prime}} \frac{\partial F_{b}}{\partial H_{\perp}},
\end{gathered}
$$

which describe the evolution of $f_{b}^{+}\left(x^{\prime}, H_{\perp}, \tau\right)$ and $f_{b}^{-}\left(x^{\prime}, H_{\perp}, \tau\right)$. In obtaining Eqs. (35) and (36) from Eqs. (32) and (34), it has been assumed that $\left[f_{b}^{+}\right]_{v=0}=$ $\left[f_{b}^{-}\right]_{v=0}$, which corresponds to the continuity of the distribution function at the turning points where $v_{x}=$ $\pm v=0$. In Eqs. (35) and (36), $v\left(H_{\perp}, x^{\prime}\right)$ is defined in Eq. (31), and $\delta \psi\left(x^{\prime}, \tau\right)$ is determined self-consistently from Eq. (33). Making use of $d p_{x}=\left(d H_{\perp}\right) / v_{x}$ and Eq. (34) to evaluate the perturbed density $\delta n_{b}\left(x^{\prime}, \tau\right)$, Poisson's equation (33) becomes

$$
\frac{\partial^{2}}{\partial x^{\prime 2}} \delta \psi=-\frac{4 \pi e_{b}^{2}}{\gamma_{b}^{2}} \int_{\psi^{0}\left(x^{\prime}\right)}^{\infty} \frac{d H_{\perp}}{v}\left(f_{b}^{+}+f_{b}^{-}\right) .
$$

Combining Eqs. (35) and (36), some straightforward algebraic manipulation gives for the evolution of $f_{b}^{+}+$ $f_{b}^{-}$,

$$
\frac{\partial^{2}}{\partial \tau^{2}}\left(f_{b}^{+}+f_{b}^{-}\right)-v \frac{\partial}{\partial x^{\prime}}\left[v \frac{\partial}{\partial x^{\prime}}\left(f_{b}^{+}+f_{b}^{-}\right)\right]=-2 v \frac{\partial}{\partial x^{\prime}}\left[v \frac{\partial \delta \psi}{\partial x^{\prime}} \frac{\partial F_{b}}{\partial H_{\perp}}\right],
$$

where $v\left(H_{\perp}, x^{\prime}\right)$ is defined in Eq. (31).

In the new variables $\left(x^{\prime}, H_{\perp}, \tau\right)$, Eqs. (37) and (38) are fully equivalent to the linearized Vlasov-Maxwell equations (27) and (28) in the original variables $\left(x, p_{x}, t\right)$. Furthermore, Eqs. (37) and (38) are applicable for small-amplitude perturbations about general equilibrium distribution $F_{b}\left(H_{\perp}\right)$. For present purposes, we specialize to the choice of stepfunction distribution in Eq. (8), which gives

$$
\frac{\partial F_{b}}{\partial H_{\perp}}=-\frac{\hat{n}_{b}}{2 \gamma_{b} m_{b} \hat{\boldsymbol{v}}_{0}} \delta\left(H_{\perp}-\hat{H}_{\perp}\right),
$$

where $\hat{\boldsymbol{v}}_{0}=\left(2 \hat{H}_{\perp} / \gamma_{b} m_{b}\right)^{1 / 2}$. Substituting Eq. (39) into Eq. (38) then gives

$$
\frac{\partial^{2}}{\partial \tau^{2}}\left(f_{b}^{+}+f_{b}^{-}\right)-v \frac{\partial}{\partial x^{\prime}}\left[v \frac{\partial}{\partial x^{\prime}}\left(f_{b}^{+}+f_{b}^{-}\right)\right]=\frac{\hat{n}_{b}}{\gamma_{b} m_{b} \hat{v}_{0}} v \frac{\partial}{\partial x^{\prime}}\left[v \frac{\partial \delta \psi}{\partial x^{\prime}}\right] \delta\left(H_{\perp}-\hat{H}_{\perp}\right) .
$$


Because the right-hand side of Eq. (40) is proportional to $\delta\left(H_{\perp}-\hat{H}_{\perp}\right)$, we look for solutions of the form

$$
f_{b}^{+}+f_{b}^{-}=g_{b}\left(x^{\prime}, H_{\perp}, \tau\right) \delta\left(H_{\perp}-\hat{H}_{\perp}\right) .
$$

We also introduce the definitions

$$
\begin{gathered}
\hat{g}_{b}\left(x^{\prime}, \tau\right) \equiv g_{b}\left(x^{\prime}, H_{\perp}=\hat{H}_{\perp}, \tau\right), \\
\hat{\boldsymbol{v}}\left(x^{\prime}\right) \equiv \boldsymbol{v}\left(H_{\perp}=\hat{H}_{\perp}, x^{\prime}\right)=\hat{\boldsymbol{v}}_{0}\left[1-\frac{\psi^{0}\left(x^{\prime}\right)}{\hat{H}_{\perp}}\right]^{1 / 2},
\end{gathered}
$$

where $\hat{v}_{0} \equiv\left(2 \hat{H}_{\perp} / \gamma_{b} m_{b}\right)^{1 / 2}$ is the speed of a particle with energy $H_{\perp}=\hat{H}_{\perp}$ as it passes through $x=0$. Substituting Eq. (41) into Eqs. (37) and (40) and integrating over $H_{\perp}$ then gives

$$
\frac{\partial^{2}}{\partial \tau^{2}} \hat{\boldsymbol{g}}_{b}-\hat{\boldsymbol{v}} \frac{\partial}{\partial x^{\prime}}\left[\hat{\boldsymbol{v}} \frac{\partial}{\partial x^{\prime}} \hat{\boldsymbol{g}}_{b}\right]=\frac{\hat{n}_{b}}{\gamma_{b} m_{b} \hat{\boldsymbol{v}}_{0}} \hat{\boldsymbol{v}} \frac{\partial}{\partial x^{\prime}}\left[\hat{\boldsymbol{v}} \frac{\partial \delta \psi}{\partial x^{\prime}}\right]
$$

and

$$
\frac{\partial^{2}}{\partial x^{\prime 2}} \delta \psi=-\frac{4 \pi e_{b}^{2}}{\gamma_{b}^{2}} \frac{\hat{g}_{b}}{\hat{v}}
$$

where $\hat{v}\left(x^{\prime}\right)$ is defined in Eq. (42), and $\delta n_{b}\left(x^{\prime}, \tau\right)=$ $\hat{g}_{b} / \hat{v}\left(x^{\prime}\right)$ is the perturbed density.

Equations (43) and (44) are coupled equations for $\hat{g}_{b}\left(x^{\prime}, \tau\right)$ and $\delta \psi\left(x^{\prime}, \tau\right)$. Substituting Eq. (44) into Eq. (43), and introducing $\delta E_{x}\left(x^{\prime}, \tau\right)=$ $-\left(\partial / \partial x^{\prime}\right) \delta \phi\left(x^{\prime}, \tau\right)=-\left(\gamma_{b}^{2} / e_{b}\right)\left(\partial / \partial x^{\prime}\right) \delta \psi\left(x^{\prime}, \tau\right)$, we obtain

$$
\hat{\boldsymbol{v}} \frac{\partial}{\partial x^{\prime}} \frac{\partial^{2}}{\partial \tau^{2}} \delta E_{x}-\hat{\boldsymbol{v}} \frac{\partial}{\partial x^{\prime}}\left[\hat{\boldsymbol{v}} \frac{\partial}{\partial x^{\prime}} \hat{\boldsymbol{v}} \frac{\partial}{\partial x^{\prime}} \delta E_{x}\right]=-\frac{\hat{\boldsymbol{\omega}}_{p b}^{2}}{\gamma_{b}^{2}} \frac{\hat{\boldsymbol{v}}}{\hat{\boldsymbol{v}}_{0}} \frac{\partial}{\partial x^{\prime}}\left(\hat{\boldsymbol{v}} \delta E_{x}\right),
$$

where $\hat{\boldsymbol{\omega}}_{p b}^{2}=4 \pi \hat{n}_{b} e_{b}^{2} / \gamma_{b} m_{b}$ is the on-axis $(x=0)$ plasma frequency squared. Equation (45) is applicable in the beam interior, $-x_{b}<x^{\prime}<x_{b}$, where $x_{b}$ is determined from $\psi^{0}\left( \pm x_{b}\right)=\hat{H}_{\perp}$ [see Eqs. (10) and (21)]. Comparing Eqs. (9) and (42), note that

$$
\frac{\hat{\boldsymbol{v}}\left(x^{\prime}\right)}{\hat{\boldsymbol{v}}_{0}}=\frac{n_{b}\left(x^{\prime}\right)}{\hat{n}_{b}}=N\left(x^{\prime}\right),
$$

where the (dimensionless) profile shape function $N\left(x^{\prime}\right)$ is defined by

$$
N\left(x^{\prime}\right)=\left[1-\frac{\psi^{0}\left(x^{\prime}\right)}{\hat{H}_{\perp}}\right]^{1 / 2} .
$$

That is, the profile shapes for $\hat{\boldsymbol{v}}\left(x^{\prime}\right)$ and $n_{b}^{0}\left(x^{\prime}\right)$ are identical, with $\hat{v}\left(x= \pm x_{b}\right)=0$ at the edges of the sheet beam. (This is expected because $\pm x_{b}$ constitute the turning points of the transverse orbits with maximum energy $H_{\perp}=\hat{H}_{\perp}$.) Equation (45) can be integrated once with respect to $x^{\prime}$, with integration constant equal to zero. The integration constant is set equal to zero, since $(\partial / \partial \tau) \delta E_{x}=0$ follows in the electrostatic approximation from the $\nabla \times \delta \vec{B}$ Maxwell equation in the vacuum region $\left(x_{b}<|x|<x_{w}\right)$ outside the layer where $\delta J_{x b}=0$. This readily gives

$$
\frac{\partial^{2}}{\partial \tau^{2}} \delta E_{x}-\hat{\boldsymbol{v}}_{0}^{2} N\left(x^{\prime}\right) \frac{\partial}{\partial x^{\prime}}\left[N\left(x^{\prime}\right) \frac{\partial}{\partial x^{\prime}} \delta E_{x}\right]=-\frac{\hat{\boldsymbol{\omega}}_{p b}^{2}}{\gamma_{b}^{2}} N\left(x^{\prime}\right) \delta E_{x},
$$

where $\hat{\boldsymbol{v}}_{0}^{2}=2 \hat{\boldsymbol{H}}_{\perp} / \gamma_{b} m_{b}$, and $N\left(x^{\prime}\right)$ is defined in Eq. (47).

Equation (48) constitutes the final dynamical equation describing the evolution of $\delta E_{x}\left(x^{\prime}, \tau\right)$ for small-amplitude perturbations about the step-function equilibrium distribution in Eq. (8). Moreover, Eq. (48) can be solved as an initial-value problem or as an eigenvalue equation (the approach used in Sec. V), and can be used to investigate detailed collective excitations over a wide range of system parameters and normalized beam intensity $s_{b}=$ $\hat{\omega}_{p b}^{2} / \gamma_{b}^{2} \omega_{\beta \perp}^{2}$.

\section{ANALYSIS OF EIGENVALUE EQUATION}

In the analysis Eq. (48), we make use of a normal-mode approach and express $\delta E_{x}\left(x^{\prime}, \tau\right)=\delta \hat{E}_{x}\left(x^{\prime}, \omega\right) \exp (-i \omega \tau)$, where $\omega$ is the (generally complex) oscillation frequency. In addition, we introduce the "stretched" spatial variable $X^{\prime}$ defined by

$$
X^{\prime}=\int_{0}^{x^{\prime}} \frac{d x^{\prime}}{N\left(x^{\prime}\right)}
$$

where $N\left(x^{\prime}\right)=n_{b}^{0}\left(x^{\prime}\right) / \hat{n}_{b}$ is defined in Eq. (47). Making use of Eq. (49) gives $N\left(x^{\prime}\right) \partial / \partial x^{\prime}=\partial / \partial X^{\prime}$, and Eq. (48) reduces to the eigenvalue equation

$$
\hat{\boldsymbol{v}}_{0}^{2} \frac{\partial^{2}}{\partial X^{\prime 2}} \delta \hat{E}_{x}+\left[\omega^{2}-\frac{\hat{\boldsymbol{\omega}}_{p b}^{2}}{\gamma_{b}^{2}} N\left(x^{\prime}\right)\right] \delta \hat{E}_{x}=0
$$

in the new variables. In Eq. (50), $N\left(x^{\prime}\right)=N\left[x^{\prime}\left(X^{\prime}\right)\right]$, where $x^{\prime}\left(X^{\prime}\right)$ is the inverse solution to Eq. (49). Note from Eq. (49) that the location of the beam edge $\left(X_{b}\right)$ in the new variables is determined from $X_{b}=\int_{0}^{x_{b}} d x^{\prime} / N\left(x^{\prime}\right)$. Equation (50) is applicable in the beam interior $\left(-X_{b}<\right.$ $X^{\prime}<X_{b}$ ) and is to be solved subject to the boundary conditions $\delta \hat{E}_{x}\left(X^{\prime}= \pm X_{b}, \omega\right)=0$. The solution of physical interest in the vacuum region in the present analysis is simply $\delta \hat{E}_{x}=0$ for $\left|X^{\prime}\right|>X_{b}$.

The eigenvalues equation (50) can be used to determine the eigenfrequency $(\omega)$ and eigenfunction $\left(\delta \hat{E}_{x}\right)$ for a specified value of normalized beam intensity $s_{b}$ and corresponding normalized density profile $N\left(X^{\prime}\right)$ (see 
Sec. III). Note that Eq. (50) is a Schrödinger-like equation with $\omega^{2}$ playing the role of energy $(E)$ and $N\left(X^{\prime}\right)$ playing the role of potential $(V)$. One general property of the eigenvalues is readily demonstrated from Eq. (50). We multiply Eq. (50) by $\delta \hat{E}_{x}^{*}\left(X^{\prime}, \omega\right)$ and operate with $\int_{-X_{b}}^{X_{b}} d X^{\prime} \cdots$. Integrating by parts with respect to $X^{\prime}$, enforcing $\delta \hat{E}_{x}\left(X^{\prime}= \pm X_{b}, \omega\right)=0$, and rearranging terms, we readily obtain

$$
\omega^{2}=\frac{\int_{-X_{b}}^{X_{b}} d X^{\prime}\left[\hat{\boldsymbol{v}}_{0}^{2}\left|\partial \delta \hat{E}_{x} / \partial X^{\prime}\right|^{2}+\left(\hat{\omega}_{p b}^{2} / \gamma_{b}^{2}\right) N\left(x^{\prime}\right)\left|\delta \hat{E}_{x}\right|^{2}\right]}{\int_{-X_{b}}^{X_{b}} d X^{\prime}\left|\delta \hat{E}_{x}\right|^{2}} .
$$

The right-hand side of Eq. (51) is manifestly real and positive, which implies that $\omega^{2}>0$. Therefore, the eigenfrequency $\omega$ is real, corresponding to purely oscillatory solutions to the eigenvalue equation (50). In particular, the absence of instability $(\operatorname{Im} \omega>0)$ for the choice of equilibrium distribution in Eq. (8) is expected because $F_{b}\left(H_{\perp}\right)$ is a nonincreasing function of energy $H_{\perp}$ [21]. Equation (51) is an exact result. If a trial function solution for $\delta \hat{E}_{x}\left(X^{\prime}, \omega\right)$ is employed, then Eq. (51) can be used to estimate the eigenvalue $\omega^{2}$.

The eigenvalue equation (50) can be used to determine the eigenfrequency $\omega^{2}$ and eigenfunction $\delta \hat{E}_{x}\left(X^{\prime}, \omega\right)$ for the entire range of normalized beam intensity, $0<$ $s_{b}<1$, where $s_{b}=\hat{\omega}_{p b}^{2} / \gamma_{b}^{2} \omega_{\beta \perp}^{2}$. We first analyze Eq. (50) in the two limiting cases corresponding to $s_{b} \rightarrow 1$ and $s_{b} \ll 1$.

\section{A. Space-charge-dominated beam $\left(s_{b} \rightarrow 1\right)$}

For a very-low-emittance, space-charge-dominated beam with $s_{b} \rightarrow 1$, the normalized density profile is $N\left(X^{\prime}\right)=n_{b}^{0}\left(x^{\prime}\right) / \hat{n}_{b}=1$ for $-x_{b}<x<x_{b}$ [see Eq. (25)], and the coordinate transformation in Eq. (49) reduces to $X^{\prime}=x^{\prime}$ and the edge location is $X_{b}=x_{b}$. In this case, $\omega^{2}-\left(\hat{\omega}_{p b}^{2} / \gamma_{b}^{2}\right) N\left(x^{\prime}\right)=\omega^{2}-\hat{\omega}_{p b}^{2} / \gamma_{b}^{2}$ is a constant in Eq. (50). The corresponding odd eigenfunctions are of the form

$$
\delta \hat{E}_{x}^{\text {odd }}=A_{n} \sin \left[(2 n+2) \frac{\pi}{2} \frac{X^{\prime}}{X_{b}}\right],
$$

and the even eigenfunctions are of the form

$$
\delta \hat{E}_{x}^{\mathrm{even}}=B_{n} \cos \left[(2 n+1) \frac{\pi}{2} \frac{X^{\prime}}{X_{b}}\right] .
$$

Here, $A_{n}$ and $B_{n}$ are constants, $n=0,1,2,3, \ldots$ is an integer, and Eqs. (52) and (53) are the solutions to Eq. (50) over the interval $-X_{b}<X^{\prime}<X_{b}$ (or equivalently, $-x_{b}<x<x_{b}$ ). Substituting Eqs. (52) and (53) into Eq. (50) gives for the eigenfrequency

$$
\omega_{m}^{2}=\frac{\hat{\boldsymbol{\omega}}_{p b}^{2}}{\gamma_{b}^{2}}+m^{2}\left(\frac{\pi \hat{\boldsymbol{v}}_{0}}{2 x_{b}}\right)^{2}
$$

where $\hat{\boldsymbol{v}}_{0}^{2}=2 \hat{H}_{\perp} / \gamma_{b} m_{b}$, and $m=2,4,6, \ldots$ for the solution in Eq. (52), and $m=1,3,5, \ldots$ for the solution in Eq. (53). Because $s_{b} \rightarrow 1$ is assumed, note that $\hat{\omega}_{p b}^{2} / \gamma_{b}^{2} \simeq$ $\omega_{\beta \perp}^{2}$ in Eq. (54). Equation (54) can also be written in the compact form $\omega_{m}^{2}=\hat{\boldsymbol{\omega}}_{p b}^{2} / \gamma_{b}^{2}+k_{m}^{2} \hat{\boldsymbol{v}}_{0}^{2}$, where $k_{m}^{2}=$ $\left(m \pi / 2 x_{b}\right)^{2}$. The eigenmodes in Eq. (54) correspond to undamped plasma oscillations, including the effects of transverse energy spread (the term proportional to $k_{m}^{2} \hat{\boldsymbol{v}}_{0}^{2}$ ) in the beam particles.

\section{B. Low-intensity beam $\left(s_{b} \ll 1\right)$}

For an emittance-dominated, low-intensity beam with $s_{b} \ll 1$, the normalized density profile is $N\left(x^{\prime}\right)=$ $n_{b}^{0}\left(x^{\prime}\right) / \hat{n}_{b}=\left(1-x^{12} / x_{b}^{2}\right)^{1 / 2}$, and the coordinate transformation in Eq. (49) reduces to $X^{\prime}=x_{b} \sin ^{-1}\left(x^{\prime} / x_{b}\right)$, or equivalently,

$$
\frac{x^{\prime}}{x_{b}}=\sin \left(\frac{\pi}{2} \frac{X^{\prime}}{X_{b}}\right),
$$

where $X_{b}=(\pi / 2) x_{b}$ is the half-thickness of the layer in the stretched variables. This readily gives $N\left(X^{\prime}\right)=$ $\cos \left(\pi X^{\prime} / 2 X_{b}\right)$, and the eigenvalue equation (50) reduces to

$$
\hat{\boldsymbol{v}}_{0}^{2} \frac{\partial^{2}}{\partial X^{\prime 2}} \delta \hat{E}_{x}+\left[\omega^{2}-\frac{\hat{\boldsymbol{\omega}}_{p b}^{2}}{\gamma_{b}^{2}} \cos \left(\frac{\pi}{2} \frac{X^{\prime}}{X_{b}}\right)\right] \delta \hat{E}_{x}=0,
$$

where $X_{b}=(\pi / 2) x_{b}$. Equation (56) has a similar form to the Mathieu equation [38] except for the boundary conditions. The normalized density profile $N\left(X^{\prime}\right)=$ $\cos \left(\pi X^{\prime} / 2 X_{b}\right)$ is found to be a good approximation to $n_{b}^{0}(x) / \hat{n}_{b}$ for values of $s_{b}$ extending as high as 0.4 .

\section{General value of normalized beam intensity $\left(0<s_{b}<1\right)$}

The eigenvalue equation (50) can also be solved numerically over the interval $-X_{b}<X^{\prime}<X_{b}$ for the general value of normalized beam intensity $s_{b}$ to determine the eigenfunctions satisfying $\delta \hat{E}_{x}\left(X^{\prime}= \pm X_{b}, \omega\right)=0$, and the corresponding eigenvalues $\omega^{2}$. Equation (50) can be represented in a convenient form by introducing the angle variable $\alpha$ defined by

$$
\alpha=\frac{\pi}{2} \frac{X^{\prime}}{X_{b}}=\frac{\omega_{0}}{\hat{v}_{0}} X^{\prime},
$$

where $X_{b}$ and $\omega_{0}$ are defined by

$$
X_{b}=\int_{0}^{x_{b}} \frac{d x^{\prime}}{N\left(x^{\prime}\right)}, \quad \omega_{0}=\frac{\pi}{2} \frac{\hat{\boldsymbol{v}}_{0}}{X_{b}},
$$

and $\hat{\boldsymbol{v}}_{0} \equiv\left(2 \hat{H}_{\perp} / \gamma_{b} m_{b}\right)^{1 / 2}$. Substituting Eq. (57) into Eq. (50) gives the eigenvalue equation

$$
\omega_{0}^{2} \frac{\partial^{2}}{\partial \alpha^{2}} \delta \hat{E}_{x}+\left[\omega^{2}-\frac{\hat{\omega}_{p b}^{2}}{\gamma_{b}^{2}} N(\alpha)\right] \delta \hat{E}_{x}=0 .
$$


Equation (59) is to be solved over the interval $-\pi / 2<\alpha<\pi / 2$ subject to the boundary conditions $\delta \hat{E}_{x}(\alpha=$ $\pm \pi / 2, \omega)=0$.

A closed expression for $N(\alpha)$ in Eq. (59) can be obtained by making use of Eqs. (12), (47), and (57). Substituting Eqs. (12) and (47) into Eq. (57) gives

$$
\alpha=\frac{\pi}{2} \frac{1}{X_{b}} \int_{0}^{x^{\prime}} \frac{d x^{\prime}}{\left[1-\hat{\psi}^{0}\left(x^{\prime}\right)\right]^{1 / 2}}=\frac{\pi}{2} \frac{\lambda_{D}}{X_{b}} \int_{0}^{\hat{\psi}^{0}} \frac{d \hat{\psi}^{0}}{\left[2\left(1-\hat{\psi}^{0}\right)\right]^{1 / 2}\left[\hat{\psi}^{0} / s_{b}-2 / 3+(2 / 3)\left(1-\hat{\psi}^{0}\right)^{3 / 2}\right]^{1 / 2}},
$$

where $\hat{\psi}^{0}\left(x^{\prime}\right) \equiv \psi^{0}\left(x^{\prime}\right) / \hat{H}_{\perp}$ and $\lambda_{D}^{2}=\gamma_{b}^{2} \hat{\boldsymbol{v}}_{0}^{2} / 2 \hat{\boldsymbol{\omega}}_{p b}^{2}$ [Eq. (11)]. Changing variables to $z=\left(1-\hat{\psi}^{0}\right)^{1 / 2}$ in Eq. (60) then gives

$$
\alpha=\frac{\pi}{2} \frac{\lambda_{D}}{X_{b}} 3^{1 / 2} \int_{\left(1-\hat{\psi}^{0}\right)^{1 / 2}}^{1} \frac{d z}{\left[(1-z)\left(a^{+}-z\right)\left(z-a^{-}\right)\right]^{1 / 2}}=\frac{\pi}{2} \frac{\lambda_{D}}{X_{b}} \frac{2 \times 3^{1 / 2}}{\left(a^{+}-a^{-}\right)^{1 / 2}} F\left(\arcsin \kappa^{-1}\left[\frac{1-\left(1-\hat{\psi}^{0}\right)^{1 / 2}}{a^{+}-\left(1-\hat{\psi}^{0}\right)^{1 / 2}}\right]^{1 / 2}, \kappa\right),
$$

where $a^{ \pm}$is defined in Eq. (18), $\kappa$ is defined in Eq. (20), and $F(\alpha, \kappa)$ is the elliptic integral of the first kind [38]. Equation (61) expresses $\alpha$ directly in terms of the normalized profile $N=n_{b}^{0} / \hat{n}_{b}=\left[1-\hat{\psi}^{0}\left(x^{\prime}\right)\right]^{1 / 2}$. Some algebraic manipulation gives exactly for the inverse function $N(\alpha)$,

$$
N(\alpha)=\frac{\left[1-a^{+} \kappa^{2} \operatorname{sn}^{2}\left(\frac{\alpha}{\pi} \frac{X_{b}}{\lambda_{D}}\left[\frac{a^{+}-a^{-}}{3}\right]^{1 / 2}, \kappa\right)\right]}{\left[1-\kappa^{2} \operatorname{sn}^{2}\left(\frac{\alpha}{\pi} \frac{X_{b}}{\lambda_{D}}\left[\frac{a^{+}-a^{-}}{3}\right]^{1 / 2}, \kappa\right)\right]},
$$

where $\operatorname{sn}(\beta, \kappa)$ is the Jacobi elliptic sine function [38]. In Eqs. (60)-(62), the stretched half-layer thickness $\left(X_{b}\right)$ measured in units of the Debye length $\left(\lambda_{D}\right)$ is given by

$$
\frac{X_{b}}{\lambda_{D}}=\frac{2 \times 3^{1 / 2}}{\left(a^{+}-a^{-}\right)^{1 / 2}} F\left[\arcsin \left(\kappa^{2} / a^{+}\right)^{-1 / 2}, \kappa\right] .
$$

Equation (63) should be compared with the expression for $x_{b} / \lambda_{D}$ in Eq. (21), where $x_{b}$ is the "unstretched" halflayer thickness. Note from Eqs. (57) and (61)-(63) that $\alpha \rightarrow \pm \pi / 2$ and $N(\alpha= \pm \pi / 2) \rightarrow 0$, as $X \rightarrow \pm X_{b}$ and $\hat{\psi}^{0}=\psi^{0} / \hat{H}_{\perp} \rightarrow 1$, as expected.

Using the expression for $N(\alpha)$ in Eq. (62), the eigenvalue equation (59) can be solved numerically for $\hat{E}_{x}(\alpha, \omega)$ and the eigenvalues $\omega^{2}$ subject to the boundary conditions $\hat{E}_{x}(\alpha= \pm \pi / 2, \omega)=0$. An approximate expression for the eigenvalues of the Schrödinger-like equation (59) can be obtained in the WKB approximation. The Born-Zommerfeld formula [39], when applied to Eq. (59), gives

$$
\frac{\hat{\boldsymbol{\omega}}_{p b}}{\gamma_{b} \omega_{0}} \int_{-\pi / 2}^{\pi / 2} d \alpha\left[\left(\frac{\gamma_{b} \omega_{m}}{\hat{\boldsymbol{\omega}}_{p b}}\right)^{2}-N(\alpha)\right]^{1 / 2}=\pi m,
$$

where $\omega_{m}$ is the $m$ th-mode eigenfrequency with $m$ halfwavelength oscillations of $\delta \hat{E}_{x}$ over the layer thickness. Making use of Eq. (61), the result in Eq. (64) can be rewritten as

$$
\begin{aligned}
6^{1 / 2} \int_{0}^{1} \frac{d z\left[q_{m}^{2}-z\right]^{1 / 2}}{\left[(1-z)\left(a^{+}-z\right)\left(z-a^{-}\right)\right]^{1 / 2}}=\frac{2 \times 6^{1 / 2}}{\left[\left(q_{m}^{2}-1\right)\left(a^{+}-a^{-}\right)\right]^{1 / 2}}\left\{\left(a^{+}-1\right) \Pi\left[\arcsin \left(\kappa^{2} a^{+}\right)^{-1 / 2}, \kappa^{2}, r\right]\right. \\
\left.+\left(q_{m}^{2}-a^{+}\right) F\left[\arcsin \left(\kappa^{2} a^{+}\right)^{-1 / 2}, r\right]\right\}=\pi m,
\end{aligned}
$$

where $a^{ \pm}$is defined in Eq. (18), and $q_{m}$ and $r$ are defined by $q_{m}=\omega_{m} /\left(\hat{\boldsymbol{\omega}}_{p b} / \gamma_{b}\right)$ and $r=\kappa\left[\left(q_{m}^{2}-a^{+}\right) /\right.$ $\left.\left(q_{m}^{2}-1\right)\right]^{1 / 2}$.

Equation (65) has been solved numerically for $\omega_{m}^{2}$, and the results have been compared with the numerical solutions of the eigenvalue equation (59). Typical results are illustrated in Fig. 3, where the normalized frequency spectrum $\omega_{m} / \omega_{\beta \perp}$ is plotted versus the normalized beam intensity $s_{b}=\hat{\omega}_{p b}^{2} / \gamma_{b}^{2} \omega_{\beta \perp}^{2}$ for several values of mode numbers $m=1,2,3,4$. In Fig. 3, the convention is such that there are $m$ half-wavelength oscillations of $\delta \hat{E}_{x}$ over the layer thickness. Strictly speaking, the WKB approximation is not expected to be applicable to the ground state with $m=1$. However, as one can see from Fig. 3, in the present analysis the WKB solution in Eq. (65) is found to give a very good approximation to the eigenvalues for all mode numbers $m \geq 1$ and all values of the normalized beam intensity $s_{b}$. For low-intensity, emittance-dominated beams $\left(s_{b} \ll 1\right)$, the mode frequencies $\omega_{m}$ are near multiples of the undepressed betatron frequency $\omega_{\beta \perp}$. For space-charge-dominated beams $\left(s_{b} \rightarrow 1\right)$, the density profile becomes flat, and the external focusing force nearly cancels the self-field force. In this limit, the beam is equivalent to an electrically neutral plasma with the external focusing force playing the role of the restoring force due to an immobile charge-neutralizing component in the neutral plasma. Therefore, in this limit, $\omega_{m} \rightarrow \hat{\boldsymbol{\omega}}_{p b} / \gamma_{b}$, where $\hat{\boldsymbol{\omega}}_{p b}=$ $\left(4 \pi \hat{n}_{b} e_{b}^{2} / \gamma_{b} m_{b}\right)^{1 / 2}$ is the relativistic plasma frequency.

Finally, in the present analysis, we have used the dimensionless parameter $s_{b}=\hat{\omega}_{p b}^{2} / \gamma_{b}^{2} \omega_{\beta \perp}^{2}$ as a measure of normalized beam intensity, where $\hat{\boldsymbol{\omega}}_{p b}=$ $\left(4 \pi \hat{n}_{b} e_{b}^{2} / \gamma_{b} m_{b}\right)^{1 / 2}$ is the relativistic plasma frequency, 


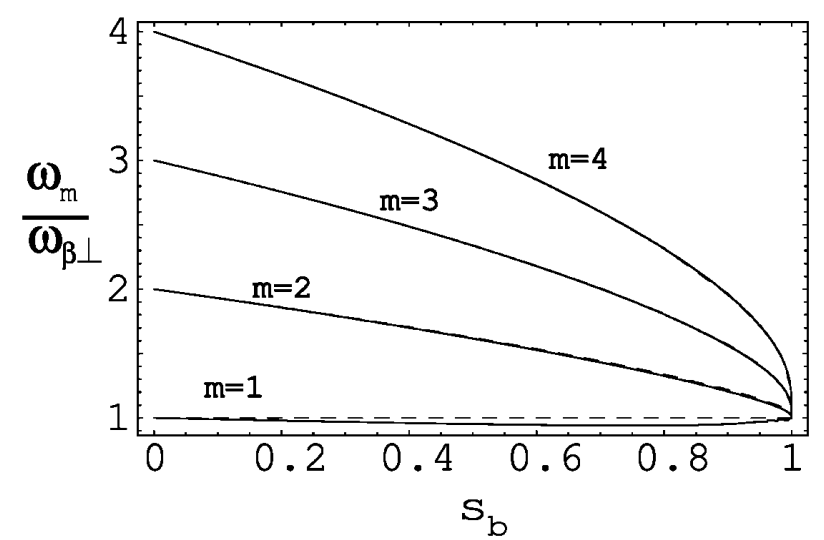

FIG. 3. Plots of the normalized mode frequencies $\omega_{m} / \omega_{\beta \perp}$ versus normalized beam intensity $s_{b}$ for several values of mode numbers $m=1,2,3,4$. The dotted curves are the numerical solutions of the eigenvalue equation (59); the solid curves are the solutions obtained in the WKB approximation [Eq. (65)].

and $\hat{n}_{b}=n_{b}(x=0)$ is the on-axis number density. It is also convenient in accelerator applications to introduce an effective tune depression $\nu / \nu_{0}$ as a measure of beam intensity. Of course, the density profile $n_{b}^{0}(x)$ in Eq. (9) is spatially nonuniform over the beam cross section, and there is a corresponding spread in (depressed) transverse betatron oscillation frequencies. For present purposes, we define the effective on-axis $(x=0)$ tune depression as the ratio of the transverse particle oscillation frequency in the presence of the beam, $\omega_{\text {osc }}=\left[\left(\gamma_{b} m_{b}\right)^{-1} \partial^{2} \psi^{0} / \partial x^{2}\right]^{1 / 2}$, to the transverse particle oscillation frequency in the applied focusing field $\left(\omega_{\beta \perp}\right)$. Making use of Eq. (7), we readily obtain for the on-axis tune depression

$$
\frac{\nu}{\nu_{0}}=\frac{1}{\omega_{\beta \perp}}\left(\omega_{\beta \perp}^{2}-\frac{\hat{\omega}_{p b}^{2}}{\gamma_{b}^{2}}\right)^{1 / 2},
$$

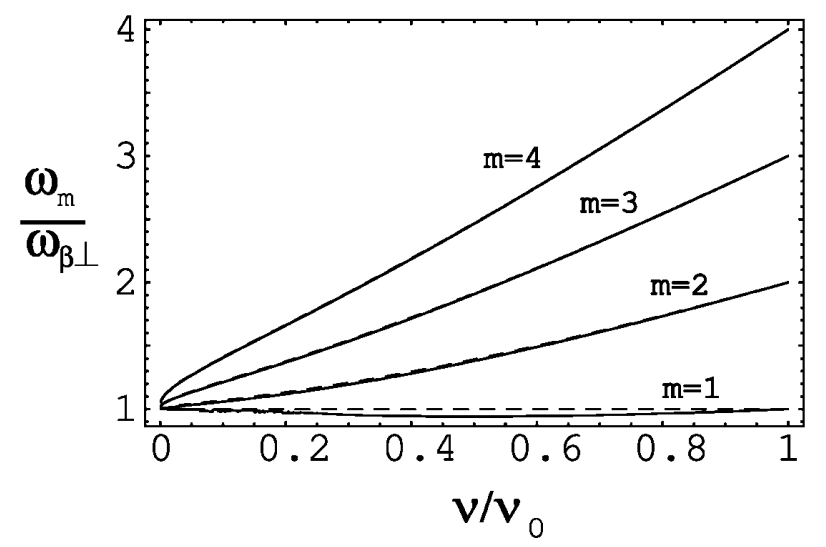

FIG. 4. Plots of the normalized mode frequencies $\omega_{m} / \omega_{\beta \perp}$ versus the on-axis $(x=0)$ tune depression $\nu / \nu_{0}=\left(1-s_{b}\right)^{1 / 2}$ for several values of mode numbers $m=1,2,3,4$ (identical conditions to those in Fig. 3). The dotted curves are the numerical solutions of the eigenvalue Eq. (59); the solid curves are the solutions obtained in the WKB approximation [Eq. (65)]. or equivalently,

$$
\frac{\nu}{\nu_{0}}=\left(1-s_{b}\right)^{1 / 2}
$$

Shown in Fig. 4 are plots of the normalized mode frequencies $\omega_{m} / \omega_{\beta \perp}$ versus the on-axis tune depression $\nu / \nu_{0}=\left(1-s_{b}\right)^{1 / 2}$ for several values of mode numbers $m=1,2,3,4$. The numerical results in Fig. 4 are fully equivalent to the results presented in Fig. 3. Note that low beam intensity $\left(s_{b} \ll 1\right)$ corresponds to $\nu / \nu_{0} \rightarrow 1$, with $\omega_{m} \simeq m \omega_{\beta \perp}$, whereas the space-charge-dominated regime $\left(s_{b} \rightarrow 1\right)$ corresponds to $\nu / \nu_{0} \rightarrow 0$, with $\omega_{m} \simeq$ $\omega_{\beta \perp} \simeq \hat{\omega}_{p b} / \gamma_{b}$.

\section{CONCLUSIONS}

To summarize, we have demonstrated that the selfconsistent water bag equilibrium $f_{b}^{0}$ satisfying the steady-state $(\partial / \partial t=0)$ Vlasov-Maxwell equations is exactly solvable for the beam density $n_{b}^{0}(x)$ and electrostatic potential $\phi^{0}(x)$. In addition, we derived a closed Schrödinger-like eigenvalue equation for smallamplitude perturbations $\left(\delta f_{b}, \delta \phi\right)$ about the selfconsistent water bag equilibrium. In the eigenvalue equation, the density profile $n_{b}^{0}(x)$ plays the role of the potential $V(x)$ in the Schrödinger equation. The eigenvalue equation was investigated analytically and numerically, and the eigenfrequencies were shown to be purely real. For the case of a space-charge-dominated beam $\left(s_{b} \rightarrow 1\right)$, the eigenfunctions and eigenfrequencies were calculated explicitly. For general values of the normalized beam intensity $0<s_{b}<1$, the WKB approximation was employed to determine the approximate expressions for the frequency spectrum as a function of the normalized beam intensity $s_{b}=\hat{\boldsymbol{\omega}}_{p b}^{2} / \gamma_{b}^{2} \omega_{\beta \perp}^{2}$, where $\hat{\boldsymbol{\omega}}_{p b}^{2}=$ $4 \pi \hat{n}_{b} e_{b}^{2} / \gamma_{b} m_{b}$, and $\hat{n}_{b}=n_{b}(x=0)$ is the on-axis number density of beam particles.

\section{ACKNOWLEDGMENTS}

This research was supported by the U.S. Department of Energy.

[1] R. C. Davidson and H. Qin, Physics of Intense Charged Particle Beams in High Energy Accelerators (World Scientific, Singapore, 2001), and references therein.

[2] A.W. Chao, Physics of Collective Beam Instabilities in High Energy Accelerators (John Wiley \& Sons, Inc., New York, 1993).

[3] M. Reiser, Theory and Design of Charged Particle Beams (John Wiley \& Sons, Inc., New York, 1994).

[4] See, for example, Proceedings of the 13th International Heavy Ion Fusion Symposium, San Diego, CA, 2000 [Nucl. Instrum. Methods Phys. Res., Sect. A 464, 1674 (2001)]. 
[5] See, for example, Proceedings of the 2001 Particle Accelerator Conference, Chicago, IL (IEEE Catalog No. 01CH37268, 2001), pp. 1-4098.

[6] R. C. Davidson, H. Qin, S. Tzenov, and E. A. Startsev, Phys. Rev. ST Accel. Beams 5, 084402 (2002).

[7] P. J. Channell, Phys. Plasmas 6, 982 (1999).

[8] R. C. Davidson, H. Qin, and P. J. Channell, Phys. Rev. ST Accel. Beams 2, 074401 (1999); 3, 029901 (2000).

[9] R. C. Davidson and H. Qin, Phys. Rev. ST Accel. Beams 4, 104401 (2001).

[10] S. I. Tzenov and R. C. Davidson, Phys. Rev. ST Accel. Beams 5, 021001 (2002).

[11] R. A. Kishek, P. G. O'Shea, and M. Reiser, Phys. Rev. Lett. 85, 4514 (2000).

[12] I. Haber, A. Friedman, D. P. Grote, S. M. Lund, and R. A. Kishek, Phys. Plasmas 6, 2254 (1999).

[13] A. Friedman, J. J. Barnard, D. P. Grote, and I. Haber, Nucl. Instrum. Methods Phys. Res., Sect. A 415, 455 (1998).

[14] S. M. Lund, J. J. Barnard, G. D. Craig, A. Friedman, D. P. Grote, T. S. Sangster, W. M. Sharp, S. Eylon, T. T. Fessenden, E. Henestroza, S. Yu, and I. Haber, Nucl. Instrum. Methods Phys. Res., Sect. A 415, 345 (1998).

[15] A. Friedman, D. P. Grote, and I. Haber, Phys. Fluids B 4, 2203 (1992).

[16] Q. Qian, W.W. Lee, and R. C. Davidson, Phys. Plasmas 4, 1915 (1997).

[17] W.W. Lee, Q. Qian, and R. C. Davidson, Phys. Lett. A 230, 347 (1997).

[18] P. Stoltz, R. C. Davidson, and W.W. Lee, Phys. Plasmas 6, 298 (1999).

[19] H. Qin, R. C. Davidson, and W.W. Lee, Phys. Rev. ST Accel. Beams 3, 084401 (2000); 3, 109901 (2000).

[20] R. C. Davidson, Phys. Plasmas 5, 3459 (1998).

[21] R. C. Davidson, Phys. Rev. Lett. 81, 991 (1998).

[22] R. C. Davidson, W.W. Lee, and P. Stoltz, Phys. Plasmas 5, 279 (1998).
[23] C. Chen and R.C. Davidson, Phys. Rev. E 49, 5679 (1994).

[24] C. Chen, R. Pakter, and R. C. Davidson, Phys. Rev. Lett. 79, 225 (1997).

[25] R. C. Davidson and C. Chen, Part. Accel. 59, 175 (1998).

[26] R. L. Gluckstern, W.-H. Cheng, and H. Ye, Phys. Rev. Lett. 75, 2835 (1995).

[27] R. C. Davidson and H. Qin, Phys. Rev. ST Accel. Beams 2, 114401 (1999).

[28] N. Brown and M. Reiser, Phys. Plasmas 2, 965 (1995).

[29] L. Struckmeier and I. Hofmann, Part. Accel. 39, 219 (1992).

[30] I. Hofmann and L. Struckmeier, Part. Accel. 21, 69 (1987).

[31] L. Struckmeier, J. Klabunde, and M. Reiser, Part. Accel. 15, 47 (1984).

[32] I. Hofmann, L. J. Laslett, L. Smith, and I. Haber, Part. Accel. 13, 145 (1983).

[33] T.-S. Wang and L. Smith, Part. Accel. 12, 247 (1982).

[34] R. L. Gluckstern, in Proceedings of the 1970 Proton Linear Accelerator Conference, Batavia, IL, edited by M. R. Tracy (National Accelerator Laboratory, Batavia, IL, 1971), p. 811.

[35] I. M. Kapchinskij and V.V. Vladimirskij, in Proceedings of the International Conference on High Energy Accelerators and Instrumentation, CERN, Geneva, 1959 (CERN Scientific Information Service, Geneva, 1959), p. 274.

[36] See, for example, Physics of Intense Charged Particle Beams in High Energy Accelerators, Chaps. 5 and 8 (Ref. [1]).

[37] R. C. Davidson, W.W. Lee, H. Qin, and E. A. Startsev, Phys. Plasmas 9, 340 (2002).

[38] I. S. Gradshteyn and I. M. Ryzhik, Tables of Integrals, Series, and Products (Academic Press, New York, 1965).

[39] L. D. Landau, Quantum Mechanics (ButterworthHeinemann, Stoneham, MA, 1997). 